\title{
Fragmentos de una historia. Un itinerario de dos décadas en el Instituto Ravignani (1956-1977)
}

Nora C. Pagano

Programa de Investigaciones en Historiografía Argentina (PIHA) del Instituto de Historia Argentina y Americana "Dr. Emilio Ravignani". Universidad de Buenos Aires-CONICET, Argentina. Correo electronico: paganonora@gmail.com

\section{Resumen}

El texto procura contribuir a la profundización del conocimiento sobre la historia de una institución dedicada al estudio del pasado argentino y americano que este año conmemora su primer centenario. Se trata un trabajo exploratorio que aspira a reconstruir algunas líneas de un itinerario institucional que se desarrolló en medio de cambiantes coyunturas políticas y sociales, entre el golpe de Estado de septiembre de 1955 hasta poco después del producido en marzo de 1976.

Tales líneas -susceptibles de ser profundizadas-, intentan dar cuenta de parte de la actividad académica desplegada en el Instituto Ravignani, tal como la misma se refleja en la documentación institucional durante la etapa sometida a análisis.

Palabras clave: Instituto Ravignani, Historia institucional, Historiografía argentina.

Fragments of a history. A two-decade itinerary at the Ravignani Institute (1956-1977)

\begin{abstract}
This work seeks to contribute to the history of the Ravignani Institute, an institution specialized in the study of Argentine and American history, which this year commemorates its first centenary. It is an exploratory work that aspires to reconstruct some lines of an institutional itinerary that developed in the midst of changing political and social conjunctures, between the coup in September 1955 until shortly after the one produced in March 1976.
\end{abstract}


These lines -susceptible to be deepened-, try to account for the academic activity carried out at the Ravignani Institute, as it is reflected in the institutional documentation, during the stage under analysis.

Keywords: Ravignan Institute, Institutional History, Argentine Historiography.

El presente texto procura contribuir a la profundización del conocimiento sobre la historia de una institución dedicada al estudio del pasado argentino y americano que este año conmemora su primer centenario. ${ }^{1}$

Se trata un trabajo exploratorio que aspira a reconstruir algunas líneas de un itinerario institucional que se desarrolló en medio de cambiantes coyunturas políticas y sociales que ilustran los agitados derroteros de la historia argentina de la segunda mitad del siglo XX. Líneas susceptibles de ser profundizadas, que intentan conocer parte de la actividad académica desplegada en el Instituto Ravignani (IR, en adelante), tal como la misma se refleja en la documentación institucional durante la etapa que abarca desde el golpe de Estado de septiembre de 1955 hasta poco después del producido en marzo de $1976 .{ }^{2}$

Corría el año 1956 y el primer número de la recientemente inaugurada segunda serie del Boletin del Instituto estaba precedido por una Advertencia de su Director-Ricardo Caillet Bois-, quien decía: "Después de un prolongado silencio de nueve años vuelve a aparecer el Boletín. Animado por los mismos principios que sustentara su fundador, Dr. Emilio Ravignani....El Instituto vuelve a tener sus puertas abiertas para todo aquel que tenga como único norte el estudio sereno, desapasionado de la verdad histórica”.

En 1980, la entonces Directora del IR, Daisy Rípodas Ardanaz, decía también en la Advertencia correspondiente al anteúltimo número -el 26- de la segunda serie del Boletín: "Este Boletín...vuelve a salir después de una involuntaria interrupción de nueve años... el Prof. Caillet-Bois, integrante de este Instituto desde la época

1 Algunos avances sobre la historia del Instituto Ravignani fueron publicados en el número 50 de Trabajos y Comunicaciones, de la Universidad de La Plata (2019). La investigación inicial se realizó en el marco de un proyecto sobre la recuperación de la memoria institucional integrado por Martha Rodríguez, María Elena García Moral, Sandra Sauro, Federico Figueiras, y quien escribe estas líneas. Dicho proyecto estuvo radicado en el programa Historia y Memoria de la Universidad de Buenos Aires dirigido por el Dr. Pablo Buchbinder. Actualmente se halla en proceso de preparación para su edición un libro que cuenta con artículos de los autores antes mencionados.

Todos los textos están basados fundamentalmente en las siguientes fuentes: Archivo Institucional Ravignani (AIR) - Archivo de la Facultad de Filosofía y Letras (AFFyL) - Boletín del Instituto (segunda serie). Respecto de éste último, hasta 1970 se publicaron con regularidad hasta los números 22-23. El número 24-25, correspondiente al período 1970-1971, fue impreso en 1973, mientras que para el número 26 -cuyos originales databan de 1978-se publicaba recién en 1980.

2 Buena parte de esas dos décadas coinciden con la prolongada gestión de Ricardo Caillet Bois como Director de esa institución (1956-73 y 1976-77). Con fines analíticos, y desde una lógica centralmente institucional, hemos subdividido esos veinte años en tres periodos: el primero abarca desde el inmediato post peronismo hasta mediados de los años '60, centrado en la reorganización/reestructuración que tuvo lugar en el Instituto y en la universidad en general. El segundo período se desarrolló desde mediados de la década del '60 hasta 1973; durante el mismo es posible verificar cierta estandarización de actividades y elencos. Finalmente una tercera estación que abarcaría hasta 1977, etapa breve pero particularmente compleja, marcada por continuidades y discontinuidades, que culmina con el regreso de Caillet Bois -renunciado desde 1973- que tuvo lugar luego del golpe de Estado del 24 de marzo de 1976. 
del Dr. Ravignami, lo dirigió con exitosa pericia — salvado un corto período por muchos conceptos omisible- desde 1955 hasta su muerte...”.

Ambas advertencias están contenidas en la segunda serie del Boletín -en tanto órgano oficial del Instituto- en el primer y en el penúltimo número de tal serie; en ellas se expresaban elocuentemente dos Directores del IR que respectivamente abren y cierran las poco más de dos décadas sometidas a análisis; ambos aludiendo a la desaparición del Boletín por espacio de casi una década; ambos recusando experiencias previas; ambos recuperando las figuras de Ravignani -en el caso de Caillet-, y de éste último por parte de Rípodas. ${ }^{3}$

Entre ambas advertencias media casi un cuarto de siglo y sin embargo los textos contienen varias similitudes conceptuales; tal circunstancia incita a la reflexión sobre las continuidades y discontinuidades de proyectos académicos desarrollados en variables contextos políticos; tiempos particularmente turbulentos durante los cuales la universidad pública -en este caso la UBA-, fue una caja de resonancia de los acontecimientos y procesos que tuvieron lugar dentro y fuera de ella.

\section{I.}

Era Ricardo Caillet Bois un discípulo cercano a Ravignani, con quien tuvo similares opciones profesionales y políticas; compartió con él espacios académicos, como por ejemplo los cursos en el Instituto del Profesorado, la Facultad de Filosofía y Letras de la Universidad de Buenos Aires (FFyL), la membresía en la Academia Nacional de la Historia (ANH) y el que nos ocupa: el ex Instituto de Investigaciones Históricas.

Consecuentemente, la cercanía política e intelectual de R. Caillet Bois con su maestro Ravignani -fallecido en 1954-, explica en parte su designación como Director del Instituto de Historia Argentina luego del golpe de Estado de 1955. Una de sus primeras medidas fue la adición a la denominación de esa institución de la expresión “Dr. Emilio Ravignani”, entendida como reconocimiento a quien fuese su primer director; ello implicaba no sólo adicionar el nombre de este último, sino preservar un espacio institucional, administrar una "herencia inmaterial", al tiempo de dar continuidad a un modo de historiar. Una de sus resultantes fue la continuación de un modelo historiográfico que Tulio Halperin Donghi definió "neutralidad erudita".

Pablo Buchbinder, en su texto sobre el Instituto en su época fundacional, señala que fue durante la gestión de Ravignani -en el contexto de la Argentina de la primera mitad del siglo XX- el momento en que tuvieron lugar buena parte de sus actividades académicas, y aun el proceso de profesionalización. 
No obstante, cabe señalar que si bien la gestión de Caillet puede considerarse como un intento de continuidad con respecto a la de Ravignani, las condiciones en que desarrolló aquella gestión fueron notoriamente distintas. En efecto, buena parte de la dirección de este último se produjo en un clima signado por la articulación entre la historiografía hegemónica y la política oficial, ambas de matriz liberal. Los años posteriores reflejaban, en cambio, las tensiones que surcaban un campo político e historiográfico diverso y acaso más complejo.

Así, por ejemplo, después del golpe de Estado de septiembre de 1955, en la FFyL y desde el punto de vista historiográfico, se producía una diferenciación entre líneas "renovadoras" y "restauradoras" (Neiburg, 1998; Rodríguez, 2019); ellas parecían expresarse en el campo académico mediante la presencia de un sector vinculado con una concepción "tradicional" de las formas de historiar, el cual coexistió con otro cuyas formas "renovadas" procuraban sintonizar a la historiografía argentina con la practicada en otros contextos. Si bien ambos grupos fueron reactivos a la experiencia política que acababa de clausurarse, la existencia de dos modelos historiográficos dotaba al campo de los estudios históricos de una particular heterogeneidad (Devoto, 1994 y 2009; Buchbinder, 1997). Ante tal configuración, la política implementada fue la distribución de espacios académicos que expresaba un complejo equilibrio de fuerzas y que proponía un clima de convivencia entre ambos sectores. ${ }^{4}$

En este marco, la hegemonía del sector "tradicional" imperante en el Instituto, será casi una constante.

Globalmente visto, el desarrollo del mismo durante esta etapa que trascurre entre 1956 - 1963 se centró en su reorganización / reestructuración a fin de obtener su reconocimiento formal por parte de la UBA, lo que implicaba estandarizar su funcionamiento y jurisdicción. ${ }^{5}$

Ello resulta perceptible en el Instituto dirigido por Caillet Bois a través de un conjunto de informes y memorias presentados fundamentalmente a partir del año 1963; mediante esos documentos es posible reconstruir una parte de la dinámica institucional, desde datos sobre la infraestructura y composición del Instituto, hasta el cambio de denominación que supuso la inclusión del área americanista.

\footnotetext{
4 Resulta pertinente señalar que por entonces se desempeñaba como decano de la FFyL el Dr. José Luis Romero, en tanto ejercía el vice decanato Luis Aznar.

En una carta del 26-5-1967, enviada por R. Caillet Bois al historiador uruguayo Eugenio Petit Muñoz agradeciéndole el envío del tercer tomo de los Documentos para la Historia de la República Oriental del Uruguay, el remitente aludía al proceso de "renovación" en estos términos "Este volumen [es] una respuesta contundente a ese nuevotipo de historiador nacido a orillas del Plata. Imbuidos de la nueva corriente de la historia social y económica, creen o simulan creer que se puede alcanzar la meta sin recurrir a los viejos y polvorientos manuscritos. Se convierten entonces en sistemáticos detractores de quienes como nosotros frecuentan la consulta de legajos en los archivos. Ellos no necesitan semejante aporte. Se valen de obras ya impresas y mediante ellas aderezan un nuevo refrito". Zubillaga (2003).
}

5 El proceso de reestructuración que también tenía lugar en la FFyL y en la misma UBA, cuya etapa inicial culminaría en 1958 con la sanción del Estatuto Universitario, en el cual se definía a los Institutos como "unidades de investigación". 
El examen de tal documentación parece fundamentar el hecho de que Caillet Bois heredó y continuó gran parte de las actividades, redes y personal que tuvieron lugar durante la gestión de Ravignani, a pesar de que, como afirmara el nuevo Director, el Instituto estaba muy disminuido en personal y recursos financieros. ${ }^{6}$ Consecuentemente puede verificarse una continuidad entre ambas gestiones, posibilitadas en gran parte gracias a un vasto conjunto de redes relacionales trazadas por Ravignani y mantenidas por el novel Director, factor que también refuerza el estilo historiográfico adoptado.

Los informes institucionales antes referidos contienen además alguna información útil acerca de investigadores e investigaciones. En tal sentido, el elenco académico posterior a 1955 vinculado al IR reconoce dos modalidades historiográficas que coinciden con dos generaciones ${ }^{7}$; los matices entre ellas se daban fundamentalmente en torno de los modos de historiar. Así, es posible observar un formato en el que predomina una historia fáctica, institucional, diplomática y de las ideas al estilo "tradicional", cercano a la Nueva Escuela Histórica. Entre ésta y la practicada por los "renovadores", había otra que, si bien no era esencialmente "renovadora", incorporaba temas y métodos vinculados con la demografía, la economía y la cuantificación, por entonces en boga. En otros términos, mientras Aznar proponía estudiar la "Historia de la FFyL y su influencia en la cultura argentina", Tjarks proyectaba abordar la "Situación económico social de la ciudadanía en Argentina, según datos de los padrones de inscripción electoral (1865-1912)", mientras que el plan de Tulio Halperin (quien no integraba el IR pero sí el Departamento de Historia y la experiencia de Historia Social) se titulaba "Economía y sociedad en Buenos Aires y su campaña (1753-1800)".

Respecto de la restructuración del Instituto -objetivo central de esta etapa-, la misma era una excelente oportunidad no sólo para acreditarlo, sino para establecer claramente sus funciones y jurisdicción, incorporando temáticas que no se restringían a la historia argentina sino que integraban a la historia americana. Se trataba de una dinámica de expansión hacia el área americanista, o más precisamente de institucionalización de la temática, ya que la misma siempre estuvo presente en el campo historiográfico local. En efecto, nuestro país fue sede de congresos americanistas desde las primeras décadas del siglo pasado; Ravignani -desde los años '20 -,y luego Caillet Bois, fueron profesores de Historia de América, la que fue también abordada por Aznar, Arocena y Torre Revello.

6 Así, por ejemplo, se inició la edición del Archivo Quiroga (iniciativa anterior a la gestión de Caillet); en materia de archivos también se comenzó con la preparación y edición del Archivo Elizalde; se continuó con la impresión de los Documentos para la Historia Argentina, y con la segunda serie del Boletin. Aquella continuidad también puede verificarse en las actividades académicas institucionales desarrolladas, como los canjes de publicaciones con instituciones similares americanas y europeas, y del personal administrativo. La disminución del personal denunciada por Caillet remite a los delegados del Instituto que revestían en el exterior -centralmente Sevilla, París, Londres y Berlín- ejerciendo tareas vinculadas con la copia de documentos.

7 Una generación más antigua constituida por Ricardo Caillet Bois (1903); Luis Aznar 1902; Julio César González (1912); Luis Arocena (1914), y otra más joven, conformada por Antonio Pérez Amuchástegui (1921); Germán Tjarks (1926). 
Las particularidades historiográficas que la inclusión de la temática americanista hacía predominantes en el Instituto consistían básicamente en vincular esa área con la historia local, de modo que ésta aparecía estrechamente relacionada con aquélla. En general, los estudios americanistas abordaban procesos, eventos y/o personajes concentrados en el periodo colonial y las primeras décadas del siglo XIX.

En ese marco, en diciembre de 1963 el Consejo Directivo (CD) de la FFyL resolvía dejar constituidos provisoriamente, a los efectos de gestionar su reconocimiento como dependencia de esta Facultad por el Honorable Consejo Superior de la UBA, a varios Institutos, entre ellos el de Historia Argentina y Americana "Dr. E. Ravignani” (IHAYA, en adelante), sobre la base del anterior Instituto de Historia Argentina “Dr. E. Ravignani”. Asimismo, el CD establecía que la dirección interina del IHAYA le correspondía a R. Caillet Bois. ${ }^{8}$

Se cerraba así una etapa importante en la historia institucional, que concluía con el nuevo plan de estudios aprobado en 1965 (Rodríguez, 2019); ese plan confirmaba la existencia de espacios diferenciados y de diversos modelos historiográficos. Sin embargo, la presencia institucional de Historia Social se diluiría aún antes del golpe de 1966; a la crisis con sus "vínculos franceses" (Devoto, 2009), habría que adicionar las turbulencias procedentes del interior de los claustros universitarios. En tal sentido, debe recordarse que en octubre de 1965 renunciaba el Decano Romero y Gino Germani había migrado a la Universidad de Harvard.

\section{II.}

Una serie de notas que van entre marzo y mediados de junio de 1966 permite conocer la composición del IR por entonces. Según las mismas, el Personal "docente o de investigación" estaba constituido por el mismo Director Caillet Bois y los profesores: A. Pérez Amuchástegui, G. Tjarks, J.C. González y L. Aznar. En materia de auxiliares de investigación rentadas, figuraban Cristina Minutolo, Margarita Montanari y Olga Garcia (de D’Agostino). En cuanto al Personal no docente, se listaba a Amalia de Caillet Bois (secretaria), M. de Nerone (auxiliar técnica) ${ }^{9}$, A Selaroff (bibliotecaria), R. Núñez (bibliotecario a cargo de fichaje y organización de la biblioteca); y asimismo se consignaba la presencia de tres mayordomos. Como un año antes, se agregaba la necesidad de rentar a los ayudantes de investigación ad honorem, a fin de intensificar las actividades de investigación, así como debía atenderse a las designaciones vencidas.

8 Resoluciones núm. 2.104 y 2.105 - 19/12/1963

La acreditación del IHAYA coincidió con la fecha en la cual, por sendas resoluciones del Consejo Directivo se creaban "Centros de Estudios" y designaban sus Directores, correspondiéndole al Centro de Estudios de Historia Social (CEHS), al Dr. José L. Romero.

Resoluciones núm, 2.106 y 2.107- 19/12/1963

9 Amalia Fanelli (luego "de Caillet Bois") y Maria de Nerone no sólo se desempeñaron en la gestión de Ricardo Caillet sino que aparecían ya citadas por Ravignani en el tomo 1 de Asambleas Constituyentes Argentinas (1937) como correctoras de pruebas de imprenta. 
Además del personal, se consignaban también "las cátedras o grupos de investigación” (sic): “Capitales, criollos e inmigrantes. 1860-1930”, dirigido por Pérez Amuchástegui; "La independencia de las colonias hispanoamericanas y la política europea”, dirigido por Caillet Bois, y el "Archivo de Elizalde”, dirigido por Tjarks.

En el informe se listaban las recepciones regulares de las publicaciones recibidas; éstas constituyen indicadores parciales de las amplias redes académicas del IR, la mayor parte precedentes de la época de la gestión anterior.

El documento suscripto por el Director terminaba expresando una necesidad "imprescindible" (subrayado en el original: la de contar con un local más amplio para albergar el repositorio). ${ }^{10}$ Personal escaso, falta de designaciones, rentas, insuficiencia edilicia: nada muy novedoso, pero si nos hemos extendido en este punto, es para mostrar la continuidad del personal académico, administrativo y de proyectos, todos los cuales permanecerán aun cuando el 28 de junio de 1966 tenga lugar el golpe de Estado que puso fin a un gobierno constitucional, luego de cierto clima anticipatorio cargado de tensiones.

En este contexto, el rector de la UBA, Hilario Fernández Long, renunció a su cargo al verse avasallada la autonomía universitaria en la Noche de los Bastones Largos, el 29 de julio de 1966. El 22 de agosto el gobierno surgido del golpe de Estado disolvió la Federación Universitaria Argentina (FUA). Las renuncias masivas de docentes no se hicieron esperar y, si bien se dieron en toda la universidad, en la FFyL ascendieron al 68,7 \% (Slemelson y otros, 1970), aunque ello pareció no afectar demasiado al personal ni a los investigadores del IR.

Si bien el archivo institucional no hay ninguna referencia directa a estas cuestiones, ciertas notas contenidas en el mismo disponían medidas que claramente referían a un nuevo orden. Para finales de 1966, ya había un Delegado del Rector de la UBA, que fue el geógrafo Horacio Difrieri, quien revestía como Profesor en la FFyL. A él se dirigía Ricardo Caillet Bois para informarlo sobre diversas cuestiones, entre las cuales se destacaba el tema Contrataciones; ciertamente la dirección del Departamento de Historia por parte de Pérez Amuchástegui resultó funcional a tales designaciones. El tema no era nuevo, pero adquirirá alguna centralidad en un medio muy burocratizado, como fue el rectorado de Luis Botet y su adopción de infructuosas medidas tendientes a "normalizar" el medio universitario.

En medio de este clima, en el IR tenían lugar dos iniciativas. Por entonces, Caillet Bois co-organizaba seis Jornadas de Métodos de Investigación de la Enseñanza de la Historia y de la Literatura Rioplatense y de los Estados Unidos, desarrolladas entre 1966 y 1971. La realización de estas Jornadas, junto con el dictado de seminarios y cursos en la Carrera de Historia de la FFyL-UBA, la actividad académica desarrollada en la Comisión Fulbright, y la oferta de becas, contribuyen a explicar la introducción y consolidación de los estudios de la historia 
de Estados Unidos en las universidades argentinas, acaso en consonancia con los cambios geopolíticos operados por entonces (Sauro, 2019).

Otro emprendimiento fue la creación en el IR de la sección de "Documentación Extranjera” en 1968; dicha sección tendría a su cargo la tarea de recopilar microfilms, fotocopias y/o copias manuscritas de documentos relativos a la historia nacional existentes en otros países, y que por lo tanto tuvieran interés para historiar el pasado local (Sauro, inédito). ${ }^{11}$ En esta empresa -que excede ampliamente el registro historiográfico-, Alemania Federal tendrá una importante gravitación ${ }^{12}$; algunos de sus resultados pueden verificarse en las publicaciones de Caillet Bois, o bien en los textos e informes realizados por Elena Rebok durante su estadía como becaria en Alemania.

En ese momento, González se desempeñaba como Director del AGN (1961-1968) (Swiderski, 2016), y Caillet Bois presidía la ANH (1970-1974) (Figueiras, inédito).

También por entonces tenía lugar la "peronización"13 de universitarios (Dip, 2017) y la experiencia de las Cátedras Nacionales (Friedemann, 2017) y Marxistas, así como la primera oleada de Centros Académicos Privados (Pagano, 2004). Buena parte del desarrollo posterior será tributario de los dos primeros factores; en este sentido, la peronización de amplios sectores de la docencia y el estudiantado, y la experiencia de las Cátedras Nacionales, fueron un antecedente valioso en tanto incluyeron en la agenda universitaria nuevos temas y nuevas perspectivas que marcaban el clima intelectual y político por fuera de la universidad. Temáticas tales como el tercermundismo, el anti-imperialismo, la revalorización de lo "nacional-popular", estuvieron revestidas de una notable centralidad; a la vez las Cátedras Nacionales fueron el primer marco institucional que dotó de cuadros dirigentes a la novel "Universidad Nacional y Popular de Buenos Aires".

III.

El triunfo en las elecciones de 1973 de la formula Perón-Perón implicó para la historia del IR la renuncia de su director, Ricardo Caillet Bois; asimismo, se abrió una breve y agitada etapa signada por la disputa entre diferentes sectores del peronismo. ${ }^{14}$

11 Según la autora, esto indicaría una cierta continuidad con la práctica de copistas y recolectores de documentos destacados en el exterior de larga data en la historia del Instituto; así, los microfilms reemplazarían a las copias manuscritas efectuadas -entre otros- por José Torre Revello.

12 Las implicancias eran también geopolíticas, periodísticas y fundamentalmente diplomáticas, tal como puede verificarse en las notas que desde la dirección del IR se cursaron a distintas embajadas, entre ellas la alemana, desde donde se donaron los primeros microfilms.

13 Con la expresión "peronización", los autores que la utilizan aluden a la incorporación al peronismo de diversos actores sociales particularmente durante la coyuntura de la resistencia peronista.

14 En las carpetas correspondientes del archivo institucional constan notas, cartas, informes, proyectos de investigación, boletines informativos que, entre otras fuentes, proporcionan una interesante información. Esas fuentes escritas fueron complementadas por otras orales -entrevistas- que, más allá de los datos que brindaron, permitieron captar climas, recuerdos, subjetividades, argumentos no hallables en 
En ese contexto, un primer periodo estuvo marcado por el predominio de la izquierda peronista, sector del que procedió un proyecto de reforma de la UBA cuyo intento de institucionalización se produjo durante el efímero rectorado de Rodolfo Puiggrós (desde el 30 de mayo al 1 de octubre de 1973). Desde ese cargo, Puiggrós instrumentó una serie de resoluciones entre las que se destaca el cambio de denominación de la institución universitaria, que pasó a llamarse Universidad Nacional y Popular de Buenos Aires (Mallimaci y Giorgi, 2007), institución concebida como "universidad del pueblo" y "al servicio de la liberación nacional".

En la FFyL el Decano Justino O' Farrell designó al frente del Departamento de Historia a Rodolfo Ortega Peña, y a Eduardo L. Duhalde como director interventor del IR, aunque en la práctica ambos compartieron la dirección del IR,institución ésta que en julio de 1973 modificó su denominación por la de Instituto de Historia Argentina y Americana "Diego Luis Molinari”. El cambio se debía a la consideración de que este último -Molinari- era más afín a la causa nacional y popular, alegando que Ravignani tuvo una "actuación pública ambigua durante la década infame".

Tras las renuncias de Duhalde y Ortega Peña en agosto de 1973, el 5 de septiembre ocuparon la co-dirección del Instituto Ana Lía Payró de Suárez y Hebe Margarita García de Bargero, en tanto la dirección del Departamento quedó a cargo de Martha Haydée Cavilliotti, secundada por Dora Ofelia Salvide.

En tal sentido es necesario marcar que la gestión en el Instituto refleja una estrecha articulación entre ambas instancias (Departamento / Instituto), fundamentalmente en lo vinculado a la comunicación, las investigaciones y la organización de seminarios; todo ello resulta perceptible a través de los boletines informativos. Eran éstos una publicación conjunta del Instituto y del Departamento de Historia, con el objeto de informar y discutir los problemas fundamentales de la carrera con estudiantes, docentes y no docentes. En el primer Boletín Informativo, fechado el 30 de noviembre de 1973, se señalaba que la investigación de la historia argentina y americana de la FFyL se canalizaría a través del Instituto Molinari, no sin cuestionar el papel que hasta entonces había cumplido la institución en tanto instrumento destinado a elaborar y difundir ideología liberal-oligárquica, dominante en el ámbito de la enseñanza oficial. El "nuevo" Instituto debía proveer de materiales y temas a las cátedras de su área, con el objeto de llevar a la práctica la asociación entre investigación y docencia, así como la elaboración de materiales interpretativos para ser utilizados en la enseñanza de la Historia en primaria y secundaria (Garcia Moral, 2018).

En cuanto al personal académico y administrativo, a través de la información institucional puede advertirse cierta coexistencia de líneas, basada en permanencias, nuevas designaciones y renuncias/exclusiones. Las permanencias marcan aquellas líneas de continuidad que generalmente se daban no a nivel de los cuadros directivos sino del resto de quienes se desempeñaba en la institución. Así, por ejemplo, a fines de 1973 revistaban como personal del Instituto Juan Severino López, Aída 
Selaroff, Liliana Inés Álvarez, Paulino R. Vázquez y Patricio Maguirre (en uso de licencia); vale señalar que tres de los cinco ya formaban parte del mismo durante la gestión de Caillet Bois.

Respecto de las nuevas designaciones, puede observarse el ingreso a estos espacios de un grupo de intelectuales bastante heterogéneo, pero fuertemente vinculado al campo nacional y popular -provenientes generalmente del peronismo y/o de las izquierdas-, para quienes la Historia era básicamente un modo de intervención política, de manera que generalmente los análisis del pasado solían vincularse con posiciones políticas de esos actores con respecto a su presente. Consecuentemente, se solicitaron por ejemplo las designaciones de Juan José Hernández Arregui y José María Rosa.

En este sentido, también podría señalarse -con las diferencias del caso-, a un grupo de docentes con trayectorias fuertemente académicas que había ingresado en los primeros '70, que no tenía un vínculo directo con el peronismo y que estaría a cargo de materias y seminarios marcados en parte por el clima de época. Varios de ellos, formados por el grupo renovador de José Luis Romero, habían regresado a la universidad en la gestión de Ángel Castellán en 1971. Ejemplos de este caso son las incorporaciones de Haydeé Gorostegui de Torres y José Luis Moreno. ${ }^{15}$

En cuanto a remociones, desplazamientos, renuncias, éstas no son masivas en la carrera de Historia y parecen constituir casos aislados (Lo Russo, 2017). Ricardo Caillet Bois, renuncia antes de que asuman las nuevas autoridades surgidas de las elecciones de 1973 (y regresará inmediatamente luego del golpe de Estado de 1976). Similar procedimiento observa Amalia Fanelli de Caillet Bois. En octubre de 1973 Julio Cesar González -docente de Historia de América II, que había reemplazado a Caillet Bois en la dirección del Departamento de Historia en los primeros meses de 1973- también renuncia a su cargo y su designación como titular de cátedra fue anulada.

Respecto de las publicaciones institucionales, resulta interesante consignar que el Boletín deja de salir por esos años, en que se cuestionaba el papel que hasta entonces había cumplido, postulándose la necesidad de una redefinición de su función y contenidos. Acaso los bruscos giros políticos impidieron la realización de los proyectos de la izquierda peronista; lo cierto es que la presidencia de Isabel Perón interrumpió dicho proceso interviniendo a la UBA y dejando cesantes a todas sus autoridades. Con la asunción de Alberto Ottalagano como rector interventor, procedente de la extrema derecha del peronismo, se asistía a la derogación de la mayor parte de las medidas tomadas en el ámbito universitario desde fines de mayo de 1973. Consiguientemente, el nuevo decano de la FFyL -Raúl Sánchez Abelenda- anulaba una gran cantidad de las medidas tomadas durante

15 Si bien ellos no formaban parte del Instituto, la referencia permite trazar un cuadro más general. Tal como consta en el legajo personal de H. Gorostegui de Torres contenido en el Archivo de la FFyL, en marzo de 1971 y por resolución 321 del Decano Ángel Castellán, se reincorporaba a la FFyL de la UBA por concurso en condición de Profesora Asociada con dedicación exclusiva en la cátedra de Historia social latinoamericana y argentina - Departamento de Historia. Un mes más tarde era designada como Directora ad honorem del CEHS. Por resolución del CD 376, del 23/7/1974, quedó virtualmente cesante. 
los decanatos de Justino O’ Farrell (1973-1974) y del efímero de Adriana Puiggrós (1974). Durante esta última gestión se había emprendido una reestructuración de los institutos y centros de estudio e investigación, en cuyo marco - por resoluciones de julio de agosto de 1974- se creó el Instituto de Estudios Argentinos y Latinoamericanos, con una serie de centros dependientes, y el Instituto de Historia Argentina y Americana había pasado a constituir uno de ellos como Centro de Estudios de Historia Argentina y Latinoamericana. Por una resolución de fines de 1974 del decano Sánchez, el Instituto fue restituido, manteniéndose el nombre "Diego Luis Molinari”, aunque anteponiéndole el cargo de "Doctor" (Mallimaci y Giorgi, 2007). Al frente de la institución fue nombrado el historiador Jorge Ocón, quien se dedicó a administrarla pero no a innovar.

En agosto de 1975 Abelenda procedió a la reestructuración de los cinco institutos -entre ellos el de Historia Argentina y Americana- colocándolos como dependencias del Departamento de Ciencias Históricas (Sección Historia). Inmediatamente después designó al frente del Instituto Molinari a Federico Ibarguren, quien renunció a poco de consumado el golpe de Estado del 24 de marzo.

En medio de un clima atravesado por conflictos y tensiones, el 12 de mayo de 1976 Ricardo Caillet Bois volvió a estar al frente del Instituto. Una verdadera "restauración” como la que había tenido lugar dos décadas antes. Ello resulta verificable a través de las primeras medidas tomadas por el director, entre las que sobresalen la vuelta a la denominación con que fuera acreditado en 1963 ("Dr. Emilio Ravignani"); la gestión que desarrolló, orientada a la reincorporación de Julio César González,y la reanudación de la publicación del Archivo de Rufino de Elizalde.

Tras el fallecimiento de Caillet Bois, el 16 de julio de 1977, fue designada como directora la profesora especializada en historia colonial Daisy Rípodas Ardanaz de Mariluz Urquijo.

En la Memoria institucional de 1977 se especificó que el personal de investigación del Instituto estaba integrado por las profesoras García de D' Agostino, Rebok y Asato, al tiempo que el personal no docente estaba integrado por el profesor López -que cumplía tareas de investigación-, Selaroff como bibliotecaria y Vázquez. En cuanto a los temas de investigación colectiva, se contaban "La conquista del desierto. De Rosas a Roca (1829-1879)", advirtiendo que estaba en su etapa heurística, y distribuida del siguiente modo: "La época de Rosas (1829-1852)", a cargo de Asato; “De Urquiza a Sarmiento (1852-1872)”, por López; y “La presidencia de Avellaneda (1874-1880)”, por García de D’ Agostino y Rebok. Se aclaraba que los investigadores mencionados habían iniciado asimismo el fichado de la documentación colonial conservada en el Instituto. Entre las investigaciones individuales concluidas se consignaban la de García de D' Agostino sobre "Aspectos de las relaciones de la República Argentina con Bolivia, Chile y Perú (1874-1879)”, la de López sobre "Las fronteras interiores y los indios en la época de la Confederación Argentina (1852-1861)", y la investigación en curso de Rebok sobre "La política alemana (aspectos económicos, políticos y culturales) en la República Argentina durante los siglos XIX y XX" (García Moral, 2019). 
De los varios informes que se hallan en la documentación institucional, los anteriormente consignados permiten conocer el perfil del personal que revestía en el IR, al tiempo que ejemplifican el tipo de registro historiográfico prevaleciente allí en la segunda mitad de los años '70.

En este último sentido, resulta clara la continuidad de un modo de historiar similar al adoptado medio siglo antes, modo que no reflejaba los cambios conceptuales, metodológicos y temáticos producidos a escala internacional. Tampoco reflejaba aquellas iniciativas producidas a nivel local -alguna de las cuales no exentas de dimensiones políticas explícitas-, que planteaban otro modelo y otra función del oficio historiográfico.

La recuperación democrática en diciembre de 1983 y la correlativa reestructuración del ámbito intelectual posibilitaron múltiples transformaciones, cuyos efectos se manifestaron en el Instituto a partir de entonces. 


\section{Bibliografía}

"Dip, N. (2017). Antecedentes y orígenes de las primeras experiencias de peronización en la UBA. 1966 - 1970. Folia Histórica del Nordeste, núm. 29, pp. 81-112.

" García Moral, M. E. (2018). De Ravignani a Molinari: el paso de la izquierda peronista por el Instituto de Historia Argentina y Americana (1973-1974). Ponencia presentada en las II Jornadas Internacionales de historia del movimiento obrero y la izquierda, organizadas por el Departamento de Historia y el Instituto de Historia Argentina y Americana "Dr. Emilio Ravignani" de la FFyL de la UBA, Buenos Aires, 3 y el 5 de octubre. (inédito)

" García Moral, M. E. (2019). De Molinari a Ravignani: el caso del Instituto de Historia Argentina y Americana (1974-1977). Trabajos y comunicaciones, núm. 50, pp. 1-11.

"Figueiras, F. (2018). La gestión de Ricardo Caillet Bois como presidente de la Academia Nacional de la Historia. Ponencia presentada en VIII Jornadas de la División Historia, Universidad Nacional de Lujan. 7 y 8 de noviembre (inédito).

" Friedemann, S. (2017). De las Cátedras Nacionales (1967-1971) a la Universidad Nacional y Popular de Buenos Aires (1973-1974). Experiencias configuradoras de institucionalidad universitaria. Sociohistórica, núm. 39, pp. 1-31.

" Lo Russo, M. B. (2017). La disputa en la aulas: la carrera de historia en la Universidad Nacional y Popular de Buenos Aires (1973-1974). Ponencia presentada en XVI Jornadas Interescuelas/Departamentos de Historia. Departamento de Historia. Facultad Humanidades. Universidad Nacional de Mar del Plata, Mar del Plata.

" Mallimaci, F. y Giorgi, G. (2007). Nacionalismos y Catolicismos en la Facultad de Filosofía y Letras de la Universidad de Buenos Aires. Ponencia presentada en VII Jornadas de Sociología. Facultad de Ciencias Sociales, Universidad de Buenos Aires, Buenos Aires.

" Pagano, N. (2019). El reordenamiento del Instituto Ravignani en los primeros '60 en la documentación institucional. Trabajos y comunicaciones, núm. 50, pp. 1-10.

" Rodríguez, Martha (2019) "Los estudios históricos en la Facultad de Filosofía y Letras (1955-1966). Planes de estudio, planteles docentes e identidades historiográficas”, en: Trabajos y comunicaciones, n. 50, pp. 1-15.

"Sauro, S. (2019)"Caillet Bois, director del Instituto y organizador de las Jornadas de métodos de investigación y enseñanza de la historia rioplatense y en Estados Unidos", Trabajos y comunicaciones, n. 50, pp. 1-16.

"Sauro, S. (2018) "La creación de la Sección de "Documentación Extranjera" en el Instituto de Investigaciones Históricas "Dr. Emilio Ravignani”". Ponencia presentada en VIII Jornadasde la División Historia, Universidad Nacional de Lujan. 7 y 8 de noviembre (inédito)

"Swiderski, Graciela (2016). La construcción del patrimonio documental en la Argentina. Actas de las Primeras Jornadas de reflexión sobre la construcción del archivo. Archivos, cultura y patrimonio, Centro de Documentación e Investigación de la Cultura de Izquierdas y Universidad Nacional de San Martín. Biblioteca Central, Buenos Aires, CeDInCl.

"Zubillaga, C. (2003).Comunidades historiográficas y renovación disciplinaria en Uruguay. Revista Complutense de Historia de América, n. 29, 2003 


\section{Fuentes institucionales}

" Archivo del Instituto "Dr. Emilio Ravignani" (AIR). Archivo de la Dirección del Instituto de Historia Argentina y Americana "Dr. Emilio Ravignani", Facultad de Filosofía y Letras, Universidad de Buenos Aires.

"Archivo de la Facultad de Filosofía y Letras (AFFyL)

"Boletín del Instituto de Historia Argentina y Americana Dr. Emilio Ravignani. Facultad de Filosofía y Letras de la Universidad de Buenos Aires. Segunda serie. 